SCHLIMME ZEITEN, BÖSE RÄUME 
Stephan Berg

\section{Schlimme Zeiten, böse Räume}

Zeit- und Raumstrukturen in der phantastischen Literatur des 20. Jahrhunderts 
CIP-Titelaufnahme der Deutschen Bibliothek

Berg, Stephan:

Schlimme Zeiten, böse Räume : Zeit- und Raumstrukturen in der phantastischen Literatur des 20. Jahrhunderts /

Stephan Berg. - Stuttgart : Metzler, 1991

ISBN 978-3-476-00746-9

ISBN 978-3-476-03350-5 (eBook)

DOI 10.1007/978-3-476-03350-5

Dieses Werk einschließlich aller seiner Teile ist urheberrechtlich geschützt. Jede Verwertung außerhalb der engen Grenzen des Urheberrechtsgesetzes ist ohne Zustimmung des Verlages unzulässig und strafbar. Das gilt insbesondere für Vervielfältigungen, Übersetzungen, Mikroverfilmungen und die Einspeicherung und Verarbeitung in elektronischen Systemen.

(C) 1991 Springer-Verlag GmbH Deutschland Ursprünglich erschienen bei J.B. Metzlersche Verlagsbuchhandlung und Carl Ernst Poeschel Verlag GmbH in Stuttgart 1991 


\section{Danksagung}

Mein Dank gilt allen denen, die dazu beigetragen haben, daß aus einer vagen, "phantastischen Idee die Wirklichkeit einer Doktorarbeit wurde.

Ganz besonderen Dank schulde ich meinen Eltern, die mir beigebracht haben, auf die wesentlichen Dinge im Leben zu achten, meinem Doktorvater Professor Erich Kleinschmidt, der dieses Arbeitsvorhaben akzeptierte, obwohl Alfred Döblin darin nicht vorkommt, Gesine für ihren unnachahmlich strahlend-blauen Optimismus, mit dem sie mich durch meine phantastischen "Zeit-Räume« begleitet hat, der Landesgraduiertenförderung Baden-Württemberg für die Sicherung meiner materiellen Existenz, meinem Computer Olivetti M24 dafür, daß er nur einmal abgestürzt ist, dem wJahrhundertsommer* ' 88 für seine sehnsüchtigen, satten, warmen Abende am Niederrimsinger Baggersee und Frau Ute Netuschil für das ıbildschöne` Manuskript. 


\section{INHALT}

Einleitung 1

\section{FORSCHUNGSDISKUSSION 4}

\section{I.1. Definitionsversuche 5}

\section{I.2. Methodendiskussion 8}

\section{I.2.1. Innerliterarische Ansätze 9}

Erwin Gradmann: Phantastik als Verwandlung - Louis Vax: Der Konflikt zwischen Realem und Möglichem - Roger Caillois: Die Problematik von Gattungsabgrenzung und Motivanalyse - Tzvetan Todorov: Die strukturalistische Kategorie der «Unschlüssigkeit - Andrzej Zgorzelski: Phantastik als Bruch der inneren litararischen Gesetze - Florian Marzin: Die Interdependenz zweier Handlungskreise

\section{I.2.2. Außerliterarische Ansätze 21}

\section{I.2.2.1. Psychoanalytische Theoriebildung 21}

Sigmund Freud: Das Unheimliche als das Verdrängte - Castex/Penzoldt: Der neurotische Autor - Weil/Seeßlen/Zondergeld: Die tabuisierte Sexualität - Joachim Metzner: Die Rückkehr zu Freud

I.2.2.2. Sozialhistorische Theoriebildung 25

Jaquemin: Aufklärung und Phantastik - J.M. Fischer: Die Bedeutung des Fin de siècle für die moderne Phantastik - Winfried Freund: Novellistik und Phantastik

\section{I.3. Kritik und Verteidigung 27}

Edmund Wilson: Phantastik als Eskapismus - Lars Gustafsson: Die reaktionäre Grundstruktur der Phantastik - Rein A. Zondergeld: Die tiefere Realität - Rabkin/Freund: Die doppelte Signatur der Phantastik: Flucht und Revolte 


\section{ZEIT- UND RAUMSTRUKTUREN IN LITERARISCHEN VORLÄUFERN DER DEUTSCHEN PHANTASTIK DES 20. JAHRHUNDERTS 39}

\section{II.1. Zeit- und Raumstrukturen in Texten der sgothic novel 40}

\section{II.1.1. Raum 40}

Der Raum als Handlungsträger in Horace Walpoles "Castle Of Ontranto Verzerrte Architektur und psychisch-physische Desorientierung - Der Raum als Objektivation der bedrohten menschlichen Befindlichkeit

\section{II.1.2. Personen 43}

Der »villain hero* und die Familie als Zentrum des Schreckens

\section{II.1.3. Zeit 45}

Die Auflösung des epischen Zeitflusses - Die Dominanz der Vergangenheit Die Tendenz zur Harmonisierung - Das wexplained supernaturalk - Der Schrekken der "gothic novelk als Ausdruck eines kompensatorischen Verlangens

II.2. Zeit- und Raumstrukturen in Texten der sSchwarzen Romantiks am Beispiel von E. T.A. Hoffmanns "Majorat* 49

\section{II.2.1. Raum 49}

"Gotische« Strukturen in der Erzählung "Das Majorat« - Die Dominanz des „bösen« Innenraums - Die Destabilisierung der Personen durch den Raum

\section{II.2.2. Zeit 53}

Die architektonisch chiffrierte Schreckensgeschichte - Dominanz der Vergangenheit, Ohnmacht der Gegenwart

\section{II.2.3. Personen 55}

Der Verlust der Handlungssouveränität - Der »allgemein« gewordene Schrecken

II.2.4. Zusammenfassender Überblick über den verzerrten Bewegungsraum bei E.T.A. Hoffmann 56

Der bürgerliche Raum als „böser« Raum - Das Fremde der Alltagswelt 
III. DIE KATEGORIE BEWEGUNGSRAUM: KULTURTHEORETISCHE ANMERKUNGEN ZUR ENTWICKLUNG VON RAUM UND ZEIT 62

\section{III.1. Der Raum und das Subjekt: Von der absoluten Gegebenheit zur subjektiven Vorstellung 62}

Der Raum als teleologische Erfüllung bei Aristoteles - Newtons unendlicher Raum - Die ontologische Notwendigkeit des absoluten Raums - Raum und Zeit als Erkenntnisprobleme - Einsteins nicht-euklidisches Raum-Zeit-Kontinuum - Die Trennung von Raum und Raumbegriff - Der Raum als symbolische Form - Der ästhetische Darstellungsraum - Der Raum als sinnstiftender Raum - Die geborgenen Räume bei Otto F. Bollnow und Gaston Bachelard

\section{III.1.1. Manifestationen des ent-sicherten Raums in Architektur und Malerei 80 \\ III.1.1.1. Architektur 80}

Die Dialektik der Architektur - Von der harmonisierenden Perspektive der Renaissance zu den (Schein)labyrinthen des Manierismus - Der Subjekt-Raum als Raumproblem - Die Entgrenzung des Raums in der Barockarchitektur Das 19. und 20. Jahrhundert - Die Verwischung von Außen- und Innenraum durch die Entwicklung der Eisenkonstruktionen - Die Auflösung der tektonischen Hierarchie - Disfunktionale Architektur: das expressionistische

\section{Konzept}

\section{III.1.1.2. Malerei 92}

Piranesis "Carcerik - Die Welt als Gefängnis - Die dämonische Perspektive Innenraum $=$ Außenraum - Der funktionslose Raum - Die Architekturvisionen Monsu Desiderios - Der unbewohnbare Raum - Die Architektur des erstarrten Augenblicks - Die photorealistische Phantastik bei Fernand Khnopff Die Verwandlung des Vertrauten in das Unheimliche

\section{III.2. Die Zeit und das Subjekt 101}

III.2.1. Das Scheitern der Geschichtsphilosophie: Von der teleologischen

Kontinuität zum bedrohten Augenblick 101

Die Genese der Geschichtsphilosophie und die Abkehr von der Chronologie Die unendliche »Progressivität» der Geschichtsphilosophie - Die Gegenwart als Leerstelle - Das Paradox der Hegel'schen Geschichtsphilosophie - Autonomiepostulat als verkappte Theodizee - Geschichte als Katastrophengeschichte - Das gefährdete Subjekt: Kierkegaard und Nietzsche - Die Abkehr von der Geschichte und die Rettung im Augenblick - Geschichte und Augen-

blick bei Walter Benjamin - Der erfültte Augenblick als endgültiges Ende 
III.2.2. Die bedrohte und die bedrohliche Erinnerung 118

Die Notwendigkeit des Vergessens bei Nietzsche - Zum Zusammenhang zwischen anwachsender Information: Geschwindigkeit und Vergessen - Gotthard Wunbergs Überlegungen zur modernen Wahrnehmung: Vergessen als Bedingung von Erinnern - Erinnerung bei Henri Bergson und Marcel Proust Vergessen als Bedingung um zu leben: Freuds These vom Reizschutz - Der Augenblick als Rettung vor Erinnern und Vergessen - Der "gute* und der »böse* Augenblick bei Kierkegaard - Walter Benjamin: Der Augenblick als "Choc*

\section{DIE ENT-SICHERUNG VON ZEIT UND RAUM IN TEXTEN DER DEUTSCHSPRACHIGEN PHANTASTIK IN DER ERSTEN HÄLFTE DES 20. JAHRHUNDERTS 131}

\section{IV.1. Die Zeit-Autoren 131}

IV.1.1. Leo Perutz: Erinnerungsverlust und Vergessenszwang 131

IV.1.1.1. Die dritte Kugel 133

Die gescheiterte Erinnerung - Erinnerungsspeicher: Partielles Gelingen, schlußendliches Scheitern - Geschichte als Schreckensgeschichte - Die phantastische Geschichte - Der «böse* Augenblick: Die Metaphorik der drei Kugeln - Brennende Erinnerung und erlösendes Vergessen

IV.1.1.2. Sankt Petri Schnee 151

Phantastik als erzählerischer Grundmodus - Die phantastische Ich-Identität Die Wirklichkeit des Traums - Der «Heldk als Zuschauer - Die monarchische und die antiquarische Geschichte

IV.1.1.3. Der Meister des jüngsten Tages 166

Das phantastische Erzählkonzept - Der Leser als Komplize des Autors - Der Detektiv als Phantast - Depersonalisation und Geschichtsangst - Die phantastische Schrift: «Ich ist ein anderer*

IV.1.2. Alexander Lernet-Holenia:

Das Scheitern der mythischen *Wiedergänger* 174

IV.1.2.1. Die Auferstehung des Maltravers 175

Der phantastische Grundkonflikt - Phantastische Kausalität und strukturelle Ambiguität - Die Funktion des phantastischen Wiedergängers (Phantastik als Modus zur Reetablierung des Vergangenen) - Vergessen und Erinnern - Der Augenblick als therapeutische Leerstelle - Paradoxie und Zwischenwelt als Schreib- und Existenzmodus 
IV.1.2.2. Der Mann im Hut 187

Zwischenwelt und phantastische Ambiguität - Clarville, der mythische Wiedergänger - Die Funktion des Hutes - Die Ambivalenz des Mythos - Die dialektische Verknüpfung von Rettung und Untergang

IV.1.2.3. Der Graf von Sain Germain 198

Die phantastische Erinnerung als „böse* Erinnerung - Das Scheitern des Subjekts an der Dominanz der Vergangenheit - Die Auflösung des Ichs - Der Untergang der Donaumonarchie als Beschleuniger der Subjektkrise - Die unrettbare Schrift

\section{IV.2. Die Raum-Autoren 205}

IV.2.1. Gustav Meyrink: Raumterror und Transzendenzsehnsucht 205

IV.2.1.1. Der Golem 205

Die phantastische Struktur des Rahmenteils als Illustration der neuzeitlichen Bewußtseinskrise - Das depersonalisierte Ich - Die Herrschaft des Raumes Raumes über das Subjekt - Der Schrecken der phantastischen Stadt - 1. Der Antiquitätenladen - 2. Das Caféhaus - Die phantastische Rettung des Subjekts - Die phantastische Lösung als Scheinrettung IV.2.2. Alfred Kubin: Der apokalyptische Raum 235

IV.2.2.1. Die andere Seite 235

Die phantastische Grundambivalenz - Die ambigue Schrift - Der Raum als Fluchtpunkt: Die Vergangenheit als Utopie - Der Raum als existentielle Bedrohung - Die böse Geschichtlichkeit des Raumes als Generator der Apokalypse Das Subjekt als halluzinierte Scheingröße

\section{SCHLUSS 255}

Anmerkungen 261

Verzeichnis der Abkürzungen 290

Literatur 291

Autorenregister 301 
*Entweder träumte ich, oder Zeit und Raum waren zur Farce geworden..

H.P. Lovecraft[1] 